\title{
Practical Limitations in Intravital Multiphoton Microscopy of the Kidney
}

\author{
P. A. Muriello, * S. G. Clendenon,* K. W. Dunn*
}

* Department of Medicine, Division of Nephrology, Indiana University School of Medicine, $950 \mathrm{~W}$. Walnut Street, R2-202 Indianapolis, IN 46202-5116, United States

Multiphoton microscopy (MPM) has become widely used by renal biologists. In fixed kidney tissue, embryonic kidneys from a mouse model of polycystic kidney disease have been studied to characterize renal development [1]. Additionally, in live rats and mice, the kidney has been externalized and imaged directly for studies of acute renal failure, microvascular leakage in renal ischemia, folic acid uptake and transport, and organic anion transport [2].

Unfortunately, imaging depth in MPM is limited to less than 200 microns in kidney tissue. This limits studies of the rat glomerulus to the Munich Wistar strain of rats which have glomeruli within 200 microns of the surface of the kidney. Fluorescence signals attenuate with depth due to light scattering and absorption in the tissue as well as from refractive index mismatch. Even in a homogenous sample, there can be refractive index mismatch between the objective lens, immersion media, coverslip, and sample which causes the focal point to broaden with depth (Fig. 1). In a heterogeneous sample, like kidney tissue, there are additional distortions from the light passing through the capsule, tubules, and glomeruli that further degrade the focus (Fig. 2).

By examining the effects of refractive mismatch in homogeneous samples and fixed kidney tissue, we are able to decrease signal attenuation with depth. Fig. 3 shows imaging depth is doubled by mounting fixed kidney in medium that reduces the mismatch caused by the heterogeneity of the tissue. Fig. 4 shows imaging depth is improved by matching the refractive index of the immersion media and mounting media (Fig. 4B), by reducing mismatch between the immersion and mounting media as well as within the tissue (Fig. 4C), and by mounting the tissue in refractive index matching medium [3] to match the refractive index of the tissue with the immersion oil (Fig. 4D).

[1] Phillips, C.L., et al., Renal cysts of inv/inv mice resemble early infantile nephronophthisis. J Am Soc Nephrol, 2004. 15(7): p. 1744-55.

[2] Molitoris, B.A., et al., Techniques to study nephron function: microscopy and imaging. Pflugers Arch - Eur J Physiol, 2009. <http://www.springerlink.com/content/m6221n553465802m>.

[3] S. G. Clendenon and M. Ferkowicz, manuscript in preparation.

[4] Acknowledgments: This work was supported by a George M. O’Brien award from the NIH (P30 DK 079312-01) and conducted at the Indiana Center for Biological Microscopy. Thanks to Ricardo Decca for the insightful discussions. 


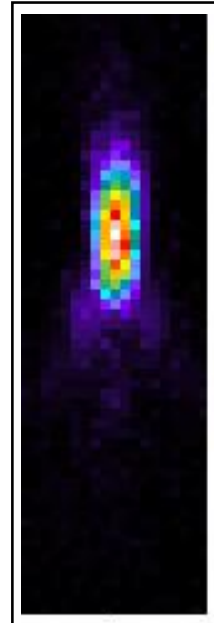

A

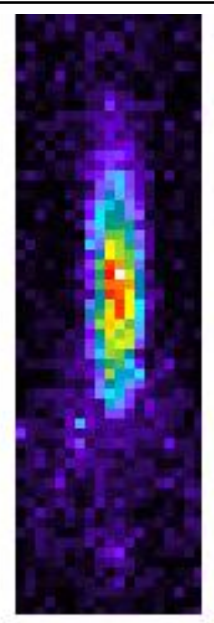

B

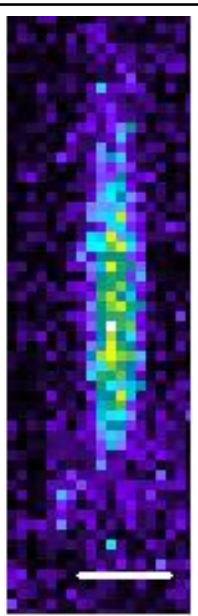

C
Fig. 1. XZ cross sectional images of 0.2 micron fluorescent microspheres mounted in agarose with refractive index, $\mathrm{n}=1.34$. Images were collected with a NA $1.460 x$ oil immersion objective (immersion oil refractive index 1.515) (A) immediately below the coverslip, (B) 20 microns deep, and (C) 50 microns deep. Scale bar is 1 micron. Pseudocolored and scaled individually for visibility.

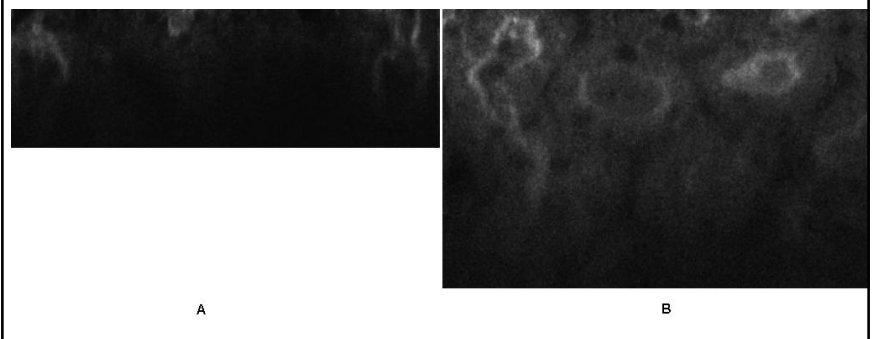

Fig. 3. XZ cross sectional image of fixed kidney tissue stained with Len CulinarisFITC. Images were collected with NA 1.2 60x water immersion objective (zoom to pixel dimension $0.3 \times 0.3$ microns.) Kidney tissue was mounted in (A) PBS and (B) 20\% PBS $80 \%$ glycerol.

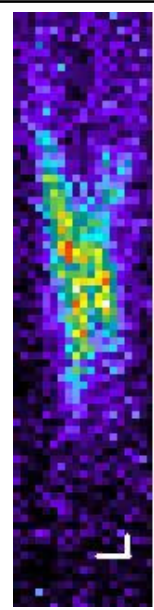

Fig. 2. XZ cross sectional image of 0.5 micron fluorescent microsphere mounted below 50 microns of fixed kidney tissue. Image was collected with NA $1.263 \mathrm{x}$ water immersion objective. Scale bars are 1 micron. Pseudocolored and scaled for visibility.
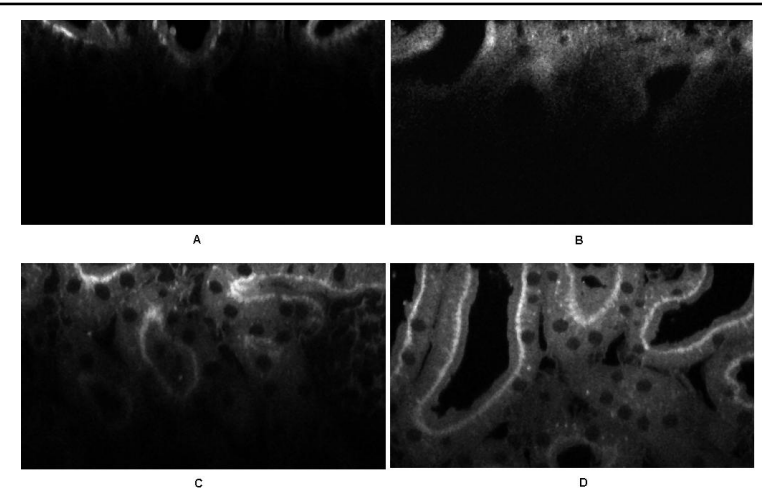

Fig. 4. XZ cross sectional images of fixed kidney tissue stained with Lens CulinarisFITC. (A) Image of tissue mounted in PBS was collected with NA $1.460 x$ oil immersion objective (oil obj.) (B) Image of tissue mounted in PBS was collected with NA 1.2 60x water immersion objective. (C) Image of tissue mounted in 20\% PBS $80 \%$ glycerol was collected with oil obj. (D) Image of tissue mounted in refractive index matching media with oil obj. All images collected with zoom to pixel dimension $0.345 \times 0.35$ microns. 